\title{
Magnetic Reconnection in Turbulent Plasmas and Gamma Ray Bursts
}

\author{
A. Lazarian* and Huirong Yan $^{\dagger}$ \\ *University of Wisconsin-Madison, USA \\ ${ }^{\dagger}$ KIAA, Peking University, China
}

\begin{abstract}
We discuss how the model of magnetic reconnection in the presence of turbulence proposed in Lazarian \& Vishniac 1999 makes the reconnection rate independent either of resistivity or microscopic plasma effects, but determined entirely by the magnetic field line wandering induced by turbulence. We explain that the model accounts for both fast and slow regimes of reconnection and that this property naturally induces flares of reconnection in low beta plasma environments. In addition, we show that the model involves volume reconnection which can convert a substantial part of the energy into energetic particles. It is important that the reconnection induces an efficient acceleration of the first order Fermi type. Finally, we relate the properties of the reconnection with the observed properties of gamma ray bursts and provide evidence supporting the explanation of gamma ray bursts based on energy release via reconnection.
\end{abstract}

Keywords: reconnection, cosmic rays, turbulence, gamma ray bursts

PACS: <Replace this text with PACS numbers; choose from this list: http: / /www.aip..org/pacs/index.html>

\section{INTRODUCTION}

Magnetic reconnection is an exciting problem of extensive history (see a book by Biskamp 2004 and references therein). Free energy associated with reconnection can power explosive events like Solar flares and gamma ray bursts. For decades, poor understanding of magnetic reconnection prevented all quantitative attempts to characterize these events. We believe that the situation is different now due to a substantial improvement of our understanding of magnetic reconnection in realistic turbulent astrophysical environments ${ }^{1}$. In particular, a model of reconnection by Lazarian \& Vishniac (1999, henceforth LV99) obtained the reconnection rate that depended only on the level of turbulence. As the model was supported by numerical testing in Kowal et al. (2009, 2012a) and the theoretical studies of the Lagrangian properties of MHD turbulence in Eyink, Lazarian \& Vishniac (2011), it became important to work on quantitative evaluations of the predictions of the LV99 theory. These predictions include the flares of magnetic reconnection that can, for instance, explain solar flares (see LV99, Lazarian \& Vishniac 2009). In addition, LV99 model entailed the first order Fermi particle acceleration during the reconnection (de Gouveia dal Pino \& Lazarian 2005). This possibility was confirmed numerically e.g. in Kowal et al. (2012b).

A possibility of LV99 model to explain gamma ray bursts was first explored in Lazarian et al. (2003). This idea was elaborated and the results were compared with in observations in Zhang \& Yan (2011).

In what follows we briefly summarize the major features of the LV99 reconnection model in §2, consider the flares that this model entails in $\S 3$, discuss the first order Fermi acceleration that induced by LV99 reconnection in $\S 4$. We consider gamma ray bursts induced by turbulent reconnection in $§ 5$. Our discussion and summary is provided in $\S 6$.

\footnotetext{
1 Turbulence is ubiquitous in astrophysics. A large number of different instabilities drive turbulence in astrophysical plasmas. In many respects the proper question is not what drives the turbulence in a particular situation, but why some reconnection models ignore the existing turbulence. In most cases, astrophysical turbulence, e.g. turbulence observed in the interstellar media is not arising from reconnection. However, in some situations, in particular, in the flares of reconnection, the reconnection itself can drive turbulence.
} 


\section{LV99 MODEL OF RECONNECTION}

\section{Extending Sweet-Parker model for turbulent fields}

Reconnection of magnetic field is driven by the free energy of magnetic field and in a generic situation of 3D geometry includes the annihilation of the component of the magnetic field that is different in the interacting magnetic fluxes. The problem of the traditional reconnection approach to the reconnection is that the most natural configuration of the reconnection presented by the Sweet-Parker model, which shown in upper part of Figure1 is very slow in astrophysical conditions. This inefficiency arises from the disparity of the astrophysical scale $L_{x}$ over which the plasma is being carried into the reconnection region and the microphysical scale $\Delta$ determined by the plasma resistivity over which the plasma is being ejected from the reconnection region. Taking into account that the ejection velocity is approximately the Alfven velocity $V_{A}$ the reconnection rate,

$$
V_{\text {rec }} \approx V_{A} \frac{\Delta}{L_{x}},
$$

is very small, $\ll V_{A}$. In fact, for the outflow region determined by the Ohmic resistivity $\Delta \approx \eta / V_{\text {rec }}$ one recovers the Sweet-Parker formulae for the reconnection rate $V_{\text {rec }, S P} \approx V_{A} R m^{-1 / 2}$, where $R m \equiv L_{x} V_{A} / \eta$, the Lundquist number, can be huge, e.g. of the order $10^{10}$ or even $10^{20}$ for many astrophysical situations. As a result, the reconnection rate in the classical Sweet-Parker model is negligible.

The situation changes dramatically in the presence of turbulence as it shown in the middle part of Figure1. Studying reconnection in the presence of turbulence LV99 showed that the outflow region is determined by the field wandering of magnetic field. Wandering or meandering of magnetic field is well known (see Jokipii 1973) and numerically tested (see Lazarian, Vishniac \& Cho 2004) effect. This effect has been used for decades to explain the perpendicular diffusion of cosmic rays in astrophysical magnetic fields.

To obtain the rate of magnetic field wandering LV99 had to extend the Goldreich-Sridhar (1995, henceforth GS95) model of strong MHD turbulence for the case of subAlfvenic turbulence ${ }^{2}$ In doing so, LV99 obtained the expressions of the magnetic reconnection for subAlfvenic turbulence:

$$
V_{\text {rec }} \approx V_{A} \min \left[\left(\frac{L_{x}}{L}\right)^{1 / 2},\left(\frac{L}{L_{x}}\right)^{1 / 2}\right] M_{A}^{2},
$$

where $M_{A} \equiv V_{L} / V_{A}$ is the Alfven Mach number and $L$ and $V_{L}$ are the turbulence injection scale and velocity at this scale, respectively.

Naturally, reconnection in the presence of sufAlfvenic turbulence provides the natural generalization of the Laminar Sweet-Parker reconnection. However, the LV99 reconnection is also applicable to transAlfvenic and superAlfvenic turbulence. The bending of magnetic field lines decreases with the decrease of the scale and at a sufficiently small scale $l_{A}=L M_{A}^{-3}$, the turbulent perturbations get subAlfvenic with LV99 model is relevant for the reconnection at this scale and smaller scales.

Finally, we should stress that the LV99 model is independent of small scale physics determining the local reconnection. LV99 conservatively assumed that the small scale reconnection happens within small scale Sweet-Parker regions (see Figure 1) and showed that this does not present a bottle-neck of the process. The key of accelerated reconnection is that many independent patches of magnetic flux reconnect simultaneously enhancing the total reconnection rate. In fact, it is possible to show (see LV99) that even with Sweet-Parker reconnection the overall reconnection rate constrained by the Ohmic diffusion exceeds $V_{A}$, which shows that the Ohmic diffusivity does not present any limitation for magnetic reconnection rate.

LV99 model also considers the motion of the bundles of reconnected flux within the reconnection zone and concludes that their interactions does not present an impediment for the reconnection rate. This also follows from a more recent paper by Eyink, Lazarian \& Vishniac (2011, henceforth ELV11) where it is shown that magnetic flux is not frozen in turbulent fluids irrespectively of their conductivity. In view of ELV11 the expression given by Eq.

\footnotetext{
${ }^{2}$ In addition, LV99 was the first paper which discussed the local system of turbulence which is one of the key ingredients of the present day understanding of MHD turbulence. The critical balance condition that is the corner stone of strong MHD turbulence is valid only in the local system of reference.
} 

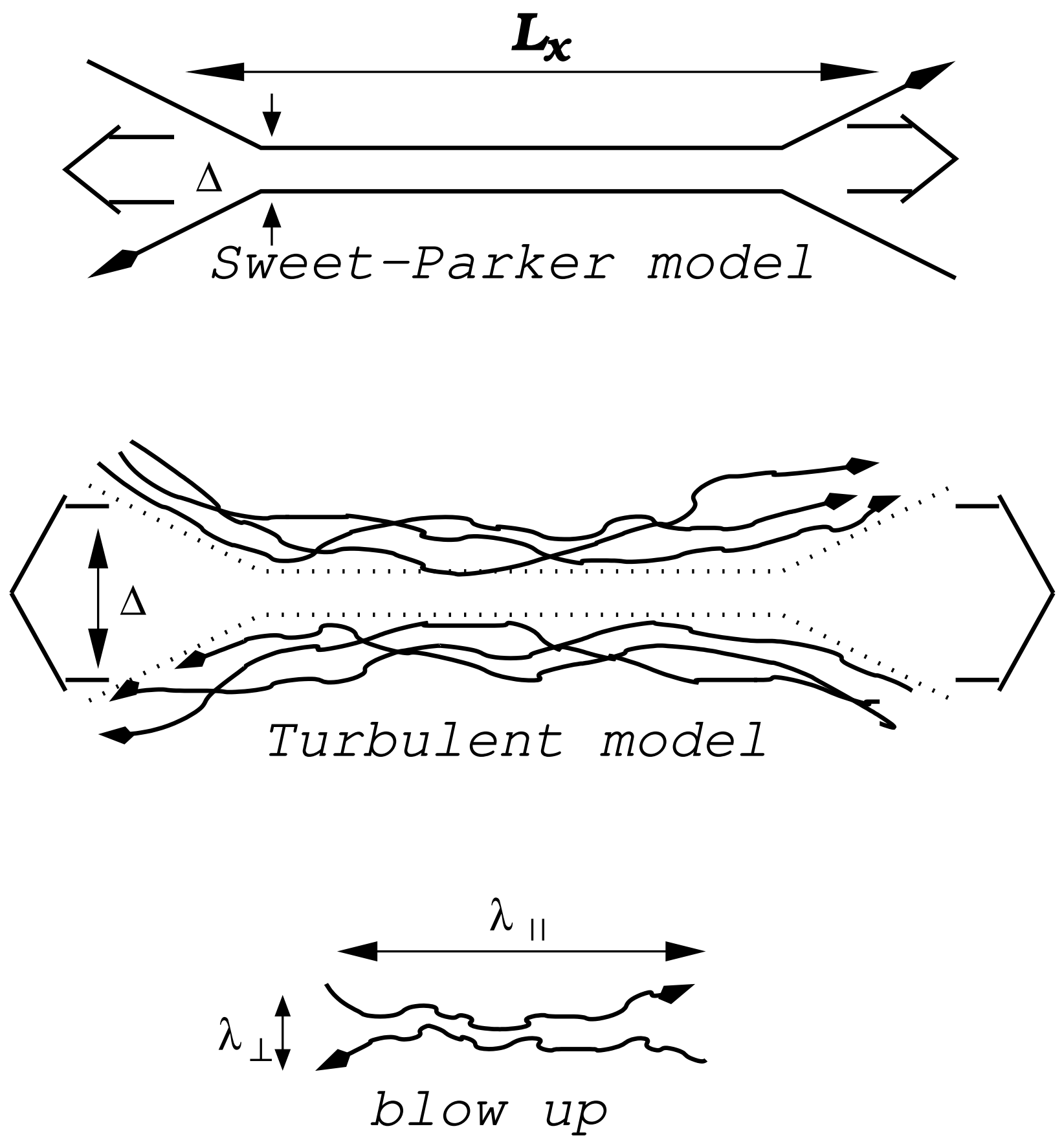

FIGURE 1. Upper plot: Sweet-Parker model of reconnection. The outflow is limited by a thin slot $\Delta$, which is determined by Ohmic diffusivity. The other scale is an astrophysical scale $L \gg \Delta$. Middle plot: Reconnection of weakly stochastic magnetic field according to LV99. The model that accounts for the stochasticity of magnetic field lines. The outflow is limited by the diffusion of magnetic field lines, which depends on field line stochasticity. Low plot: An individual small scale reconnection region. The reconnection over small patches of magnetic field determines the local reconnection rate. The global reconnection rate is substantially larger as many independent patches come together. The illustration of LV99 reconnection from Lazarian et al. 2004.

(2) is a direct consequence of this violation of frozen-in condition. This is a result of Richardson diffusion present in turbulent fluids (see Eyink 2011). The possibility of deriving the Eq. (2) from the approach different from the one used in the original LV99 treatment strengthens the theoretical foundations of the discussed extention of Sweet-Parker 

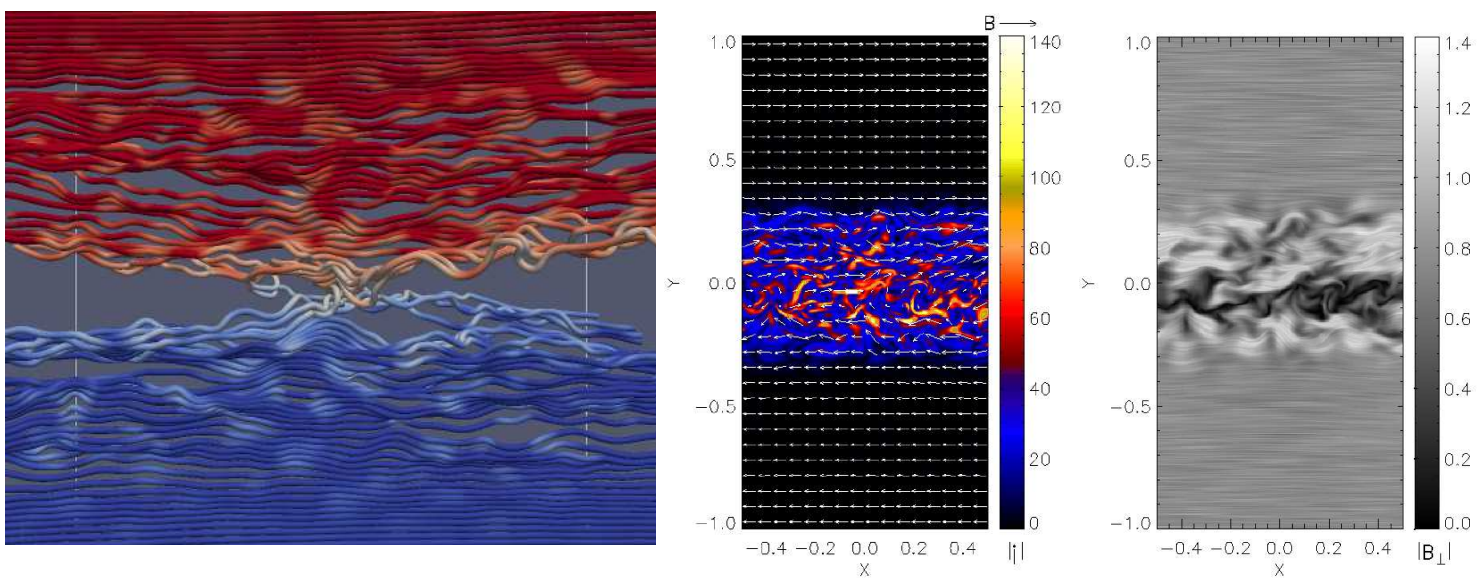

FIGURE 2. Left panel: Magnetic field in the reconnection region. Large perturbations of magnetic field lines arise from reconnection rather than driving; the latter is subAlfvénic. The color corresponds to the direction of magnetic lines. Central panel: Current intensity and magnetic field configuration during stochastic reconnection. We show a slice through the middle of the computational box in the xy plane after twelve dynamical times for a typical run. The shared component of the field is perpendicular to the page. The intensity and direction of the magnetic field is represented by the length and direction of the arrows. The color bar gives the intensity of the current. The reversal in $B_{x}$ is confined to the vicinity of $\mathrm{y}=0$ but the current sheet is strongly disordered with features that extend far from the zone of reversal. Right panel: Representation of the magnetic field in the reconnection zone with textures. From Lazarian et al. (2011).

reconnection in turbulent fluids.

\section{Numerical testing of LV99 model}

Numerical studies of reconnection is tricky as the Lundquist numbers corresponding to numerical simulations are puny compared to astrophysical one. The usual routine of increasing the resolution several times to see the effect on reconnection may not work due to this disparity. For instance, even a weak dependence of reconnection rate on the Lundquist number, which is extremely difficult to notice with the traditional "change by factor of two" approach, can make the reconnection rate completely negligible for for astrophysics, as the corresponding numbers increase by a factor of $10^{10}$ or larger. Thus it is essential that LV99 provides analytical dependences (see Eq. (2)) that can be tested numerically.

Numerical studies of LV99 model were presented in Kowal et al. (2009, 2012a). The numerical set-up was used to test 3D magnetic reconnection in the presence of guide field (i.e. magnetic field fluxes intersect at arbitrary angles) and in the presence of subAlfvenic turbulence. The reconnection was not forced and open boundary conditions were employed. Turbulence was driven both in Fourier space and in real space. Both the input power of the turbulence driving and the scale of the driving were varied to test the analytical predictions of the LV99 model.

It is illustrated in Figure 2 that the turbulence in the simulations is driven around the reconnection region. This mimics the effects of astrophysical turbulence that is known to exist in high Reynolds number astrophysical fluids ${ }^{3}$. The driving is subAlfvenic with only gentle perturbations of the magnetic field lines induced. This driving, nevertheless, results in magnetic field wandering which, in agreement with LV99 model, opens up the outflow region and induces reconnection independent of resistivity.

A comparison of results of numerical testing and theoretical predictions obtained in LV99 is shown in Figure 3. For subAlfvenic turbulence the input power $P_{i n j}$ scales as $V_{L}^{4}$. As a result the predicted reconnection rate (see Eq. 2) scales

\footnotetext{
${ }^{3}$ Volume driving that we use in the simulations corresponds well the actual driving in turbulence in astrophysical conditions, where smaller eddies get energy from the larger ones. This driving results in homogeneous balanced turbulence as opposed to inhomogeneous imbalanced one resulting from the driving induced at the numerical box boundaries. A theoretical description of such turbulence is currently absent and predictions of the reconnection rates for such turbulence is currently impossible.
} 


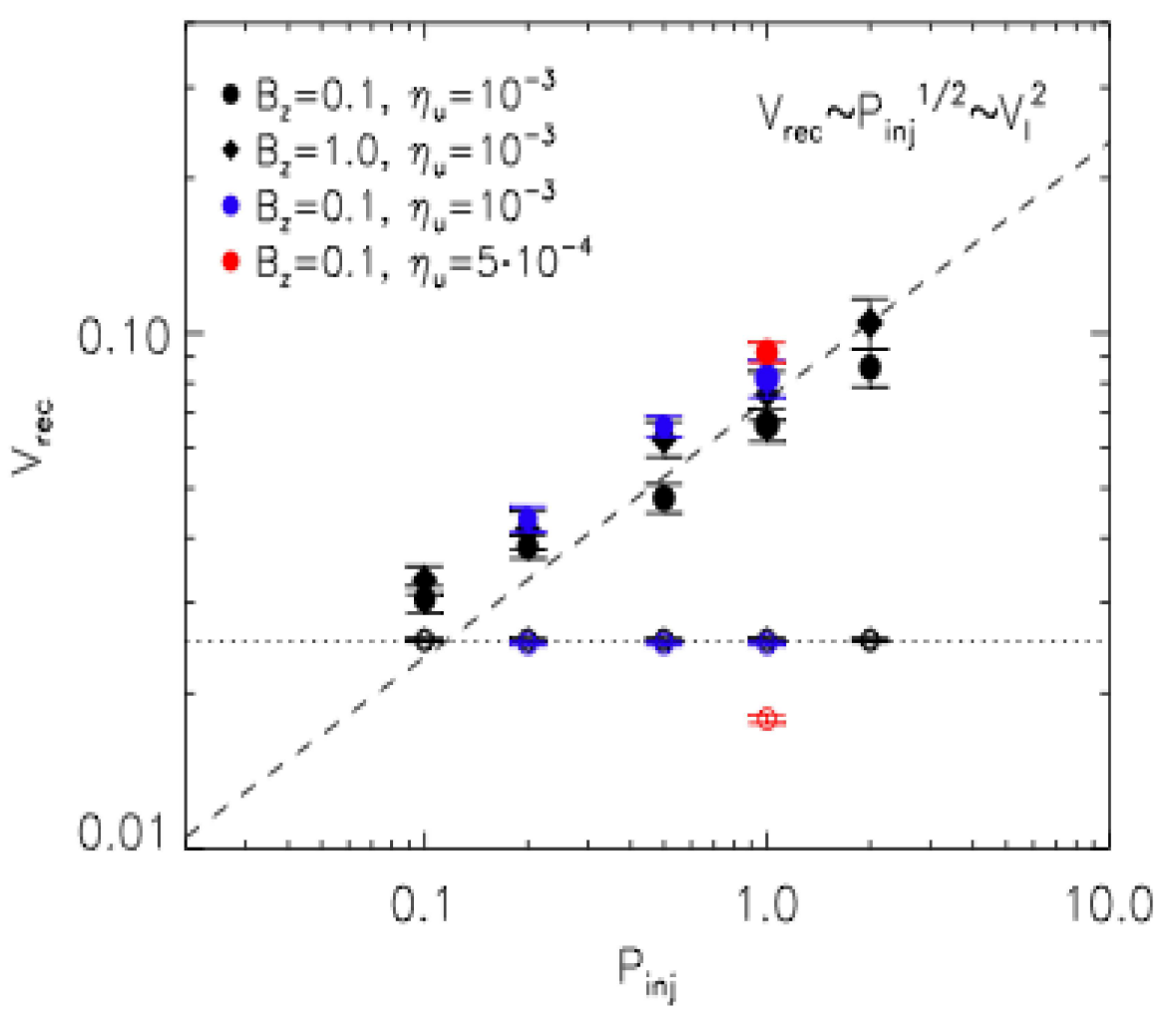

FIGURE 3. Reconnection speed versus input power $P_{i n j}$ for the driven turbulence. Left: Variations of reconnection speed in time for different levels of turbulent driving Right: Reconnection speed, plotted against the input power for an injection wavenumber equal to 8 (i.e. a wavelength equal to one eighth of the box size) and a resistivity $v_{u}$. The dashed line is a fit to the predicted dependence of $P^{1 / 2}$. The horizontal line shows the laminar reconnection rates for each of the simulations before the turbulent forcing started. From Kowal et al. (2012a).

as $V_{\text {rec }} \sim P_{i n j}^{1 / 2}$. It is easy to see that this dependence is in good agreement with the results of numerical simulations. In Figure 3 the uncertainties in the time averages are indicated by the size of the symbols and the variances are shown by the error bars. The higher resolution $1028 \times 512 \times 512$ simulations are shown with red color. Those corresponds to lower Sweet-Parker rate, but the rate in the presence of turbulence does not change.

For decades the it has been considered that plasma effects, e.g. Hall effect ${ }^{4}$, are essential for making magnetic reconnection fast. The use of anomalous resistivity, i.e. the non-linear resistivity that depends on the value of the current density is one of the accepted ways to simulate plasma effects. Figure 4 shows that the reconnection rate does not change with the anomalous resistivity in the presence of turbulence. As expected, the reconnection rate of turbulent magnetic field does not depend on the Ohmic resistivity either. This confirms that the LV99 reconnection is fast.

\section{Relation to other ideas}

LV99 is a volume reconnection and it was proposed at the time when X-point Petschek reconnection dominated the landscape of reconnection ideas (see Shay et al. 1998). Therefore the observations of thick outflow regions was the evidence in favor of LV99 model. As the publication of the corresponding observational result (Cirravella \& Raymond 2008) was somewhat delayed, the competing models evolve towards more resemblance to LV99, getting

\footnotetext{
${ }^{4}$ A detailed theoretical discussion why the Hall term is subdominant in the presence of pre-existing turbulence can be found in ELV11.
} 

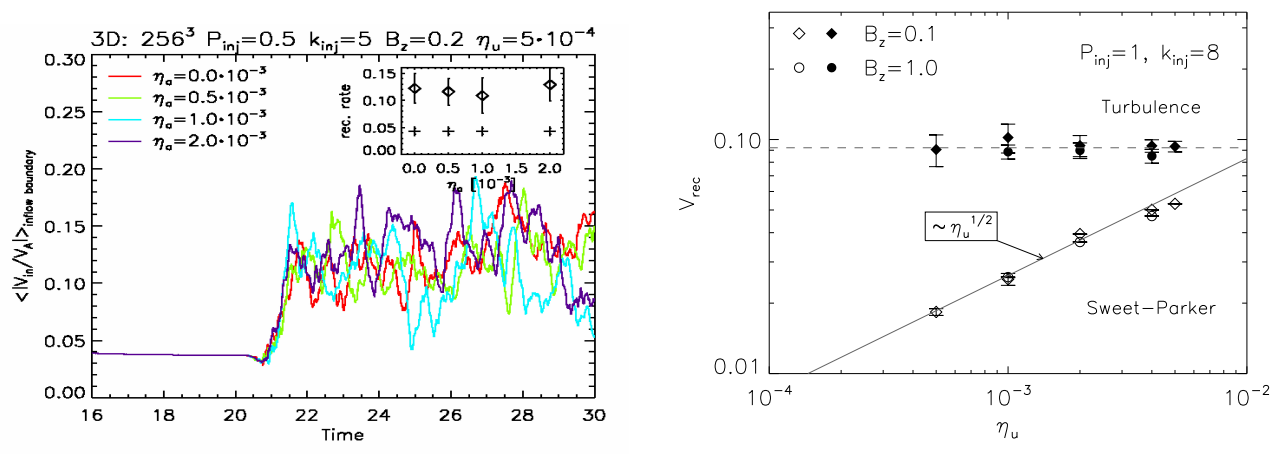

FIGURE 4. Left: Effect of anomalous resistivity on the reconnection speed. No dependence is observed. Right: No dependence on the Ohmic diffusion is observed either. The lower curve reproduces the Sweet-Parker reconnection rates for the laminar case. From Kowal et al. (2009).

chaotic structure of magnetic field and thick outflow regions. This convergence of ideas on reconnection makes it more challenging to distinguish them observationally, especially, as new modeling indicates not only tearing instability development but a transition to turbulence (see Karimabadi 2012).

The idea of tearing instability (see Loreiro et al. 2007) may be considered to be complementary to the LV99 model. The process, for instance, can lower the threshold for the reconnection bursts that we discuss in $\S 3$.

Finally, we mention, that the LV99 model is radically different from what was proposed earlier in terms of models of how turbulence can affect reconnection. For instance, in a study by Matthaeus \& Lamkin (1985, 1986), which is the closest to the spirit to LV99 the authors performed 2D numerical simulations of turbulence and provided arguments in favor of magnetic reconnection getting fast. However, the physics of the processes that they considered was very different from that in LV99. For instance, the key process of field wandering of the LV99 model has not been considered in the aforementioned papers. On the contrary, processes that they discuss, e.g. X-point reconnection and possible effects of heating and compressibility are not ingredients of the LV99 model. Other papers discussing turbulence, e.g. Speiser (1970) and Jacobson (1984) are even more distant in terms of the effects that they explored. The aforementioned authors studied the changes of the microscopic properties of the plasma induced by turbulence and considered how these changes can accelerate magnetic reconnection. On the contrary, LV99 shows that the microscopic plasma properties are irrelevant for their model of reconnection (see also Figure 4).

\section{FLARES OF RECONNECTION}

\section{Reconnection instability}

In the text above we have discussed how the pre-existing turbulence changes the nature of reconnection. The next question is what happens when the magnetic fields are initially laminar. The answer to this question in LV99 is that a reconnection instability exists and can drive reconnection in a bursty fashion. Indeed, These bursts of reconnection are easy to understand by considering low $\beta$ (i.e. highly magnetized) plasmas with magnetic laminar flux tubes coming into contact with each other. Initially, the magnetic reconnection is going to proceed at a slow pace (see Figure 1), as magnetic field lines are laminar. However, the system of two highly magnetized flux tubes in contact is unstable to the development of turbulence. Indeed, if the outflow gets turbulent, turbulence should spread over field lines of surrounding flux, inducing their wandering and extending the width of the outflow region $\Delta$ and increasing the energy injection in the system via the increase of $V_{r e c}$. Both factors increase the level of turbulence in the system ${ }^{5}$ inducing a positive feedback leading to an explosion of reconnection.

A characteristic feature of this reconnection instability is that it is finite amplitude instability and therefore it can allow the accumulation of the flux prior to reconnection. In other words, LV99 model predicts that the reconnection

\footnotetext{
${ }^{5}$ For instance, the increase of $\Delta$ increases the Reynolds number of the outflow, making the outflow more turbulent.
} 
can be both fast and slow, which is the necessary requirement of bursty reconnection frequently observed in nature, e.g. in Solar flares. This process may be related to the bursts of reconnection observed in simulations in Lapenta (2008). In addition, LV99 predicted the process of triggered reconnection when reconnection in one part of the volume sends perturbations that initiate reconnection in adjacent volumes. Such process was reported recently in the observations of Sych et al. (2009).

All in all, in the absence of external turbulence, the original outflow, e.g. originated through tearing instability (see Loureiro et al. 2007, Bhattacharjee et al. 2009), gets turbulent and triggers the mechanism described above. This shows that the tearing and turbulent mechanisms may be complementary ${ }^{6}$. We believe that such a mechanism can be important for explaining a wide variety of astrophysical processes ranging from solar flares to gamma ray bursts as well as bursty reconnection in the pulsar winds.

\section{A Simple model}

A simple quantitative model of flares was presented in Lazarian \& Vishniac (2009). It can be understood by considering a reconnection region of length $L_{x}$ and thickness $\Delta$. The thickness is determined by the diffusion of field lines, which is in turn determined by the strength of the turbulence in the volume. Reconnection will allow the magnetic field to relax, creating a bulk flow. However, since stochastic reconnection is expected to proceed unevenly, with large variations in the current sheet, we can expect that some unknown fraction of this energy will be deposited inhomogeneously, generating waves and adding energy to the local turbulent cascade. This is a natural outcome of the turbulent flow

We take the plasma density to be approximately uniform so that the Alfven speed and the magnetic field strength are interchangeable. The nonlinear dissipation rate for waves is

$$
\tau_{\text {nonlinear }}^{-1} \sim \max \left[\frac{k_{\perp}^{2} v_{\text {wave }}^{2}}{k_{\|} V_{A}}, k_{\perp}^{2} V L\right]
$$

where the first rate is the self-interaction rate for the waves and the second is the dissipation rate by the ambient turbulence (see Beresnyak \& Lazarian 2008). The important point here is that $k_{\perp}$ for the waves falls somewhere in the inertial range of the strong turbulence. Eddies at that wavenumber will disrupt the waves in one eddy turnover time, which is necessarily less than $L / V_{A}$. The bulk of the wave energy will go into the turbulent cascade before escaping from the reconnection zone.

We can therefore simplify our model for the energy budget in the reconnection zone by assuming that some fraction $\varepsilon$ of the energy liberated by stochastic reconnection is fed into the local turbulent cascade. The evolution of the turbulent energy density per area is

$$
\frac{d}{d t}\left(\Delta V^{2}\right)=\varepsilon V_{A}^{2} V_{r e c}-V^{2} \Delta \frac{V_{A}}{L_{x}},
$$

where the loss term covers both the local dissipation of turbulent energy, and its advection out of the reconnection zone. Since $V_{\text {rec }} \sim v_{\text {turb }}$ and $\Delta \sim L_{x}\left(V / V_{A}\right)$, we can rewrite this by defining $M_{A} \equiv V / V_{A}$ and $\tau \equiv L_{x} / V_{A}$ so that

$$
\frac{d}{d \tau} M_{A}^{3} \approx \varepsilon M_{A}-M_{A}^{3}
$$

If $\varepsilon$ is a constant then

$$
V \approx V_{A} \varepsilon^{1 / 2}\left[1-\left(1-\frac{M_{A}^{2}}{\varepsilon}\right) e^{-2 \tau / 3}\right]^{1 / 2} .
$$

This implies that the time during which reconnection rate rises to $\varepsilon^{1 / 2} V_{A}$ is comparable to the ejection time from the reconnection region $\left(\sim L_{x} / V_{A}\right)$. Given that reconnection events in the solar corona seem to be episodic, with longer periods of quiescence, this indicates that either $\varepsilon$ is very small, for example, dependent on the ratio of the thickness of the current sheet to $\Delta$. If it scales as $M_{A}$ to some power greater than two then initial conditions dominate the early time evolution.

\footnotetext{
${ }^{6}$ When turbulence develops the LV99 mechanism can provide much faster reconnection compared to tearing and tearing may become a subdominant process. In fact, emerging turbulence may suppress the tearing instability.
} 
Another route by which stochastic reconnection might be self-sustaining would be in the context of a series of topological knots in the magnetic field, each of which is undergoing reconnection. The problem is sensitive to geometry, however. We assume that as each knot undergoes reconnection it releases a characteristic energy into a volume which has the same linear dimension as the distance to the next knot. The density of the energy input into this volume is roughly $\varepsilon V_{A}^{2} V / L_{x}$, where $\varepsilon$ is the efficiency with which the magnetic energy is transformed into turbulent energy. We have

$$
\varepsilon \frac{V_{A}^{2} V}{L_{x}} \sim \frac{v^{\prime 3}}{L_{k}},
$$

where $L_{k}$ is the distance between knots and $v^{\prime}$ is the turbulent velocity created by the reconnection of the first knot. This process will proceed explosively if $v^{\prime}>V$ or

$$
V_{A}^{2} L_{k} \varepsilon>V^{2} L_{x}
$$

This condition is easy to fulfill. The bulk motions created by reconnection will generate significant turbulence as they interact with their surrounding, so $\varepsilon$ should be of order unity. Moreover the length of any current sheet should be at most comparable to the distance to the nearest distinct magnetic knot. The implication is that each magnetic reconnection event will set off its neighbors, boosting their reconnection rates from $V_{L}$, set by the environment, to $\varepsilon^{1 / 2} V_{A}\left(L_{k} / L_{x}\right)^{1 / 2}$ (as long as this is less than $V_{A}$ ). The process will take a time comparable to $L_{x} / V_{L}$ to begin, but once initiated will propagate through the medium with a speed comparable to speed of reconnection in the individual knots. In a more realistic situation, the net effect will be a kind of modified sandpile model for magnetic reconnection in the solar corona and chromosphere. As the density of knots increases, and the energy available through magnetic reconnection increases, the chance of a successfully propagating reconnection front will increase.

\section{LV99 MODEL AND PARTICLE ACCELERATION}

As any model of fast reconnection, LV99 model transfers only a small fraction of energy into heat via Ohmic heating. Most of the energy ends up in the motion of magnetic fields and plasmas. Turbulent outflow naturally suggests the possibility of the second order Fermi acceleration of energetic particles. However, this is not the only process of acceleration.

Analyzing the dynamics of the volume reconnection that occurs within LV99 outflow zone, de Gouveia dal Pino \& Lazarian (2005, henceforth GL05, see also Lazarian 2005) showed that the first order Fermi acceleration should take place as the energetic particles bounce between approaching mirrors and follow the contracting loops of magnet flux (see Figure 5).

The acceleration of energetic particles in contracting loops shown in left plot of Figure 5 is analogous to the acceleration proposed later in an influential paper by Drake et al. (2006). The difference is that the latter paper appealed to magnetic islands produced by collisionless reconnection. However, in any realistic 3D configuration of reconnecting magnetic field, 2D islands are improbable and 3D magnetic loops should be considered instead. The difference between the nature of LV99 and collisionless reconnection is not important as far as the reconnection process is concerned. Both processes increase the parallel component of the bouncing particle and difference between treatments in GL05 and Drake et al. (2006) is that the latter paper took into account the possible effects of feedback via firehose instability on the particle acceleration.

In addition to the acceleration of terms of the parallel momentum, the first order Fermi acceleration in terms of perpendicular momentum is also possible (see Figure 5, Right). There the particle with a large Larmor radius is bouncing back and forth between converging mirrors of reconnecting magnetic field systematically getting an increase of the perpendicular component of its momentum. Both processes take place in reconnection layers. Both processes of the acceleration in terms of parallel and perpendicular momentum are observed in the numerical studies of magnetic reconnection in the presence of turbulence as it is shown in Kowal et al. (2012a). Figure 6 illustrates the acceleration process that takes place in the turbulent reconnection layer. It is easy to see that both parallel and perpendicular momenta increase in the process.

The first order acceleration arising from the reconnection of turbulent astrophysical magnetic fields was invoked to explain the origin of anomalous cosmic rays in Lazarian \& Opher (2009) (see also Drake et al. 2010) and cosmic ray anisotropies in Lazarian \& Desiati (2010). A discussion of more cases of the acceleration via reconnection is surely to follow. For instance, as we alluded earlier, the acceleration within the reconnection regions that separate of spirals of pulsar winds is very promising. Reconnection is also invoked for the acceleration of cosmic rays in galaxy 

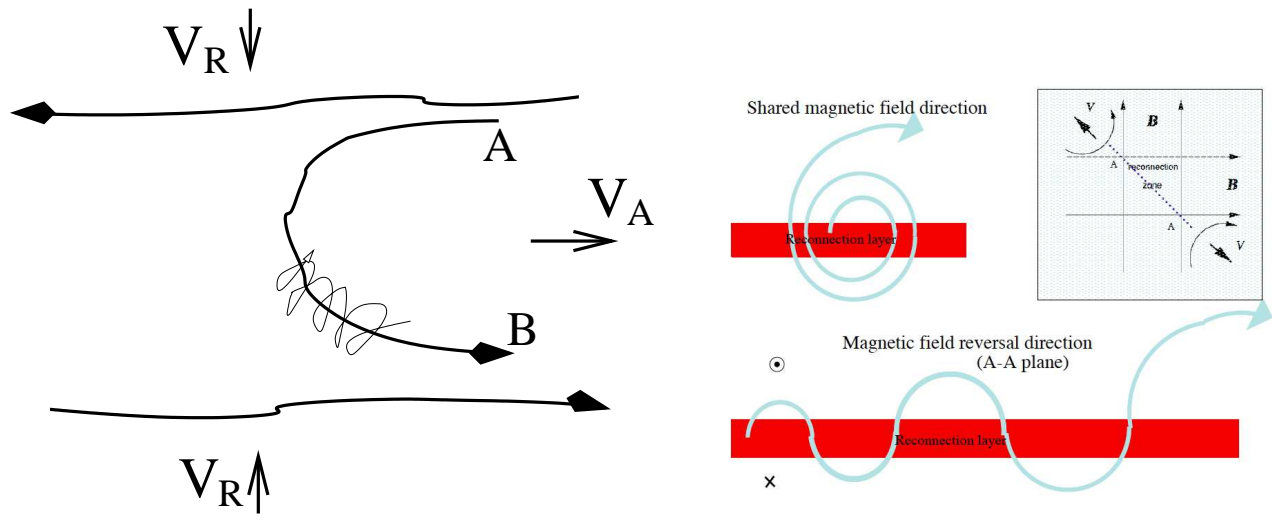

FIGURE 5. Left Cosmic rays spiral about a reconnected magnetic field line and bounce back at points A and B. The reconnected regions move towards each other with the reconnection velocity $V_{R}$. From Lazarian 2005. Right: Particles with a large Larmor radius gyrate about the magnetic field shared by two reconnecting fluxes (the latter is frequently referred to as "guide field". As the particle interacts with converging magnetized flow corresponding to the reconnecting components of magnetic field, the particle gets energy gain during every gyration.
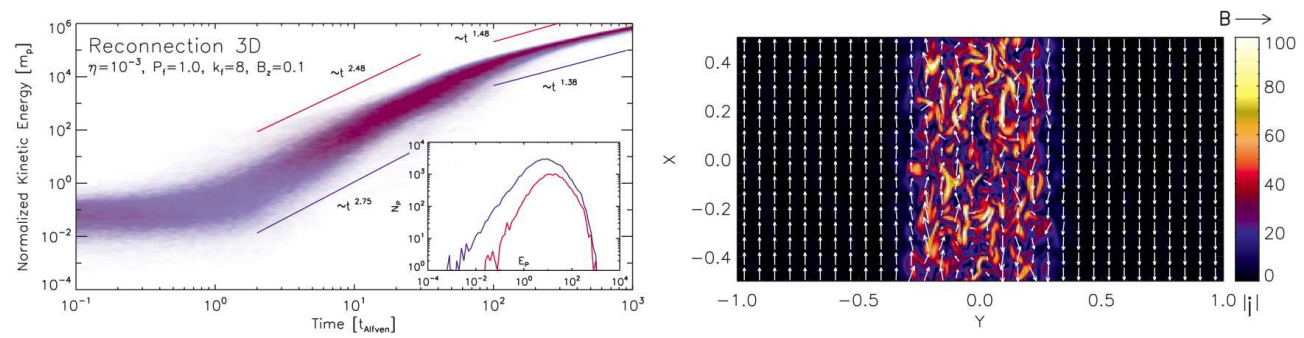

FIGURE 6. Left Acceleration of energetic particles in magnetic reconnection in the presence of turbulence. Red color corresponds to the preferential acceleration in terms of the parallel momentum, blue corresponds to the perpendicular momentum increase. Right: Reconnection layer corresponding to the process of the acceleration. From Kowal et al. 2012.

clusters (Brunetti \& Lazarian 2012). In some cases the acceleration is considered within loops of contracting magnetic field without direct reference to turbulent reconnection (see Hoshino 2011). However, as turbulence is ubiquitous in astrophysical environments it is difficult to avoid its crutial influence on reconnection (see Figure 5, right). Moreover, as we discussed in $\S 3$, in magnetically dominated environments, i.e. in the environments where turbulent reconnection is important, the reconnection is bound to induce turbulence. Below we discuss the energization of particles through reconnection that results in gamma ray bursts.

\section{APPLICATION TO GAMMA RAY BURSTS}

\section{Problem of Gamma Ray Bursts}

New questions on Gamma Ray Bursts (GRBs) were proposed thanks to Swift and Fermi observations. In particular, recent Fermi observation of GRB 080916C indicates that the bright photosphere emission associated with a putative fireball is missing, which is challenging to the traditional fireball internal shock (IS) model. A Poynting-flux-dominated outflow from the central engine is more preferable at least for this burst.

In the standard fireball IS scenario (Pacýnski et al. 1994; Shemi et al. 1990), magnetic fields are not important, i.e. $\sigma<<1$, where $\sigma$ is the ratio between the Poynting flux and the matter (baryonic + leptonic) flux. An alternative picture is that the magnetic field is dynamically important in GRB outflows, i.e. $\sigma>>1$. In this case GRB radiation would be powered by the magnetic field energy in the ejecta (see, e.g., Usov 1992; Thompson 1994; Lazarian et al. 2003; Lyutikov \& Blandford 2003). The analogy with AGNs jets also implies that magnetic dominated jets as a viable 

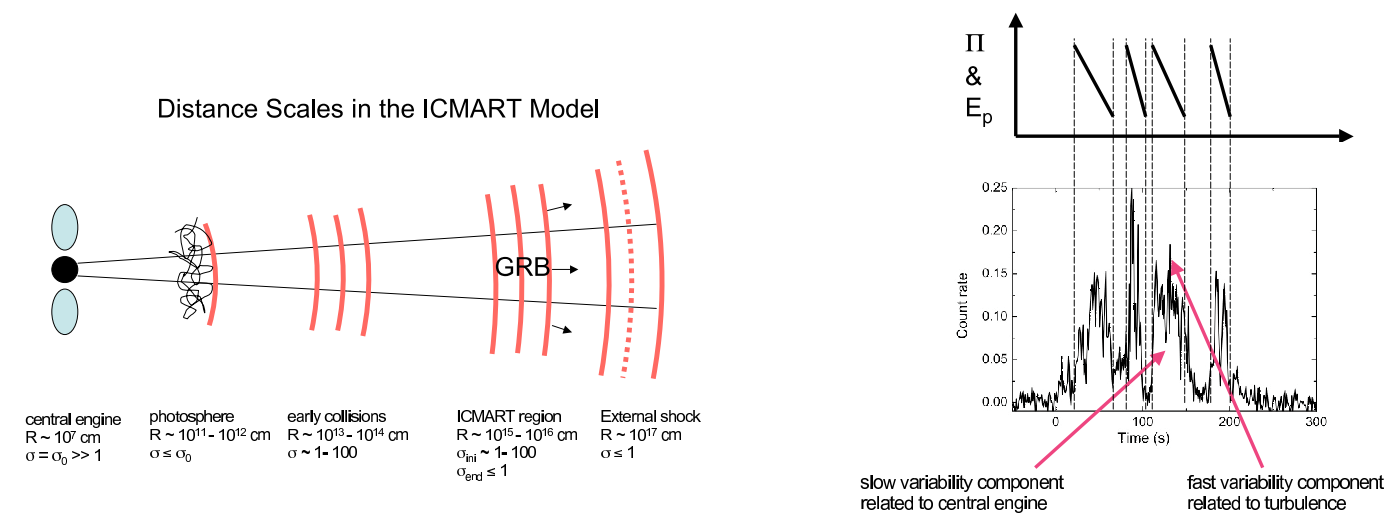

FIGURE 7. Left panel: A cartoon picture of the ICMART model. The typical distances and $\sigma$ values of various events are marked. Right panel: An example of GRB light curve that shows two variability time scales. The light curve of GRB 050607 is taken from the NASA Swift GRB archival data web site http://swift.gsfc.nasa.gov/docs/swift/archive/grb_table/ grb_lookup.php?grb_name=050607. The predictions of decreasing gamma-ray polarization degree $\Pi$ and the spectral peak energy $E_{p}$ within individual pulses are indicatively presented. Detailed decaying functions would be different depending on the details of evolution of magnetic field configuration, $\sigma$ value, as well as balance between heating and cooling of electrons. The general decreasing trend is robust. (from Zhang \& Yan 2011)

option for the source of GRBs. It has become clear that the inner engine cannot accelerate baryonic dominated jets for AGNs, suggesting that similarly GRBs are Poynting flux dominated at least in its inner region the jet.

\section{LV99 and GRBs}

Magnetic reconnection has been suggested as the engine of GRBs. The problem lay, however, in the intrinsic difficulty of reconnection since it is a very slow process in ordered fields. As with the case for solar flares, both a slow phase and a fast bursty phase are required for reconnection. Essential progress was made by Lazarian et al. (2003), where a new GRB model of self-adjusted reconnection was proposed based on the LV99 reconnection ${ }^{7}$. As the turbulence builds up the reconnection rate increases, which induces a positive feedback, resulting in the explosive reconnection. This can be an alternative scenario to gamma ray bursts (Lazarian et al. 2003). More recently the model was elaborated and connected with observational data in (Zhang \& Yan 2011).

As in the IS model, the mini-shells interact internally at the radius $R_{\mathrm{IS}} \sim \Gamma^{2} c \Delta t$. Most of these early collisions, however, do not trigger any magnetic reconnection, instead only lead to perturbations of the magnetic field in the ejecta. As in other astrophysical environment, the system is prone to turbulence because of high Reynolds and magnetic Reynolds numbers ${ }^{8}$ Since GRBs outflow is highly relativistic, the magnetic field is unlikely to be totally distorted. In any case, fast reconnection can be triggered in weakly stochastic magnetic field (LV99). The details require further studies especially in the regime of relativistic turbulence.

The perturbations accumulate as the mini-shells propagate outward (see Fig.7 right) till a point where the turbulence reaches the threshold to trigger a fast reconnection. Fast reconnection events rapidly eject outflows, which makes the system even more turbulence. This leads to a run-away release of the magnetic field energy in a reconnection/turbulence avalanche. This is one ICMART event, corresponding to one GRB pulse. The $\sigma$ value decreases to

\footnotetext{
${ }^{7}$ Indeed LV99 was proposed as a model of reconnection in non-relativistic case. However, the scaling and anisotropies of magnetized turbulence were found to be the same for both relativistic and non-relativistic cases (Cho \& Lazarian 2012). Since turbulence properties are the most important ingredient that determines the reconnection rate in LV99 model, it is natural to assume that the reconnection is similar in relativistic and nonrelativistic cases. In the former case, we expect the reconnection velocity to approach the velocity of light, as $V_{A} \rightarrow c$.

${ }^{8}$ This is a nontrivial statement since the system is highly collisionless. Nonetheless, due to the high magnetization, most kinetic motions are perpendicular to magnetic field so that it is the much suppressed perpendicular viscosity and resistivity that should be adopted.
} 
order of unity from the original value during this process.

A GRB consists of several ICMART events (i.e. broad pulses). The peak energy $E_{p}$ is expected to drop across each pulse. The $\gamma$-ray polarization degree is also expected to fall from $\sim 50-60 \%$ to $\sim$ a few $\%$ during each ICMART event. There should be two variability components in the GRB light curves, one slow component associated with the central engine activity, and a fast component related to the relativistic magnetic turbulence (see Fig.7). More details can be found in Zhang \& Yan (2011).

\section{DISCUSSION AND SUMMARY}

Turbulence is a natural state of astrophysical fluids that are characterized by very high Reynolds numbers. Therefore it should be taken into account for various astrophysical processes and magnetic reconnection is not an exception. In fact, in particular cases when the level of turbulence in the astrophysical systems is low, which can be the case of low $\beta$ plasmas, the reconnection itself induces turbulence resulting in reconnection instability and bursts of reconnection as we discussed in $\S 3$.

LV99 model provides analytical predictions for the rate of reconnection in the presence of magnetic field and those predictions have been successfully tested numerically in Kowal et al. $(2009,2012 a)$. In addition, the necessity of the LV99 reconnection for explaining the properties of MHD turbulence and making MHD turbulence self-consistent was established both in LV99 and ELV11. The model can also account for observed properties of solar flares, e.g. bursts of reconnection, triggering of reconnection, fast reconnection in collisional media etc. This makes it advantageous to apply the model to describe reconnection in various astrophysical contexts.

The energy released within LV99 reconnection can efficiently accelerate particles via both the first order Fermi acceleration and the second order Fermi acceleration. This can power gamma ray busts. The observations are consistent with the theoretical predictions and this calls for more elaborate modeling of reconnection associated with the gamma ray bursts.

\section{ACKNOWLEDGMENTS}

A.L. research is supported by the NASA Grant NNX09AH78G, as well as the support of the NSF Center for Magnetic Self-Organization. The Humboldt Award at the Universities of Cologne and Bochum, as well as Vilas Associate Award are acknowledged. H. Y. acknowledges the support from NSFC Grant AST -11073004 and the visiting professorship from IIP, Natal, Brazil.

\section{REFERENCES}

1. Bhattacharjee, A., Huang, Y.-M., Yang, H., \& Rogers, B. 2009, Physics of Plasmas, 16, 112102

2. Biskamp, D. 2003, Magnetohydrodynamic Turbulence, CUP

3. Brunetti, G. \& Lazarian, A. 2012, MNRAS, submitted

4. Cho, J. \& Lazarian, A. 2012, ApJ, in preparation

5. Ciaravella, A., \& Raymond, J. C. 2008, ApJ, 686, 1372

6. de Gouveia dal Pino, E. M., \& Lazarian, A. 2005, Astron. Astrophys., 441, 845

7. Drake, J. F., Swisdak, M., Che, H., \& Shay, M. A. 2006, Nature, 443, 553

8. Drake, J. F., Opher, M., Swisdak, M., \& Chamoun, J. N. 2010, ApJ, 709, 963

9. Eyink, G. L. 2011,Phys. Rev. E 83, 056405

10. Eyink, G. L., Lazarian, A., \& Vishniac, E. T. 2011, ApJ, 743, 51 (ELV11)

11. Jacobson, A. R., \& Moses, R. W. 1984, Phys. Rev. A, 29, 3335

12. Jokipii, J. R. 1973, ApJ, 183, 1029.

13. Goldreich, P. \& Sridhar, S. 1995, ApJ, 438, 763

14. Kowal, G., de Gouveia Dal Pino, E., \& Lazarian, A. 2012, Phys. Rev. Lett.

15. Kowal, G., Lazarian, A., Vishniac, E. T., \& Otmianowska-Mazur, K. 2012, Nonlinear Processes in Geophysics, 19, 297

16. Kowal, G., Lazarian, A., Vishniac, E. T., \& Otmianowska-Mazur, K. 2009, ApJ, 700, 63

17. Lapenta, G. 2008, Phys. Rev. Lett., 100, 235001

18. Lazarian, A. 2005, Magnetic Fields in the Universe: From Laboratory and Stars to Primordial Structures., 784,42

19. Lazarian, A. 2006, ApJL, 645, L25

20. Lazarian, A., Kowal, G., Vishniac, E., \& de Gouveia Dal Pino, E. 2011, Planetary and Space Science, 59,537 
21. Lazarian, A., \& Desiati, P. 2010, ApJ, 722, 188

22. Lazarian, A., \& Opher, M. 2009, ApJ, 703, 8

23. Lazarian, A., \& Pogosyan, D. 2004, ApJ, 616, 943

24. Lazarian, A., \& Vishniac, E. T. 2009, Revista Mexicana de Astronomia y Astrofisica Conference Series, 36, 81

25. Lazarian, A. \& Vishniac, E. 1999, ApJ, 517, 700 (LV99)

26. Lazarian, A., Vishniac, E. \& Cho, J. 2004, Astrophys. J., 603, 180

27. Lazarian, A., Vlahos, L., Kowal, G., Yan, H., Beresnyak, A., de Gouveia Dal Pino E. 2012, Space Science Review, DOI 10.1007/S 11214-9934-7

28. Loureiro, N. F., Schekochihin, A. A., \& Cowley, S. C. 2007, Physics of Plasmas, 14, 100703

29. Matthaeus, W. H., \& Lamkin, S. L. 1985, Physics of Fluids, 28, 303

30. Matthaeus, W. H., \& Lamkin, S. L. 1986, Physics of Fluids, 29, 2513

31. Paczýnski, B., \& Xu, G. 1994, Astrophys. J., 427, 708.

32. Petschek, H.E. Magnetic field annihilation. The Physics of Solar Flares, AAS-NASA Symposium (NASA SP-50), ed. WH. Hess (Greenbelt, MD: NASA) 425

33. Shay, M. A. \& Drake, J. F., 1998, Geophys. Res. Letters Geophysical Research Letters, 25, 3759-3762

34. Shemi, A., \& Piran, T. 1990, Astrophys. J. Lett., 365, L55.

35. Speiser, T. W. 1970, Planetary Space Science, 18, 613

36. Sych, R., Nakariakov, V. M., Karlicky, M., \& Anfinogentov, S. 2009, Astron. Astrophys., 505, 791

37. Thompson, C. 1994, Mon. Not. R. Astron. Soc., 270, 480.

38. Usov, V. V. 1992, Nature, 357, 472.

39. Uzdensky, D. A. \& Kulsrud, R. M. 2006, Physics of Plasmas, 13, 062305

40. Uzdensky, D. A., Loureiro, N. F. and Schekochihin, A. A. 2010. Phys. Rev. Lett.105, 235002.

- Zhang, B. \& Yan, H. 2011, ApJ, 726, 90 


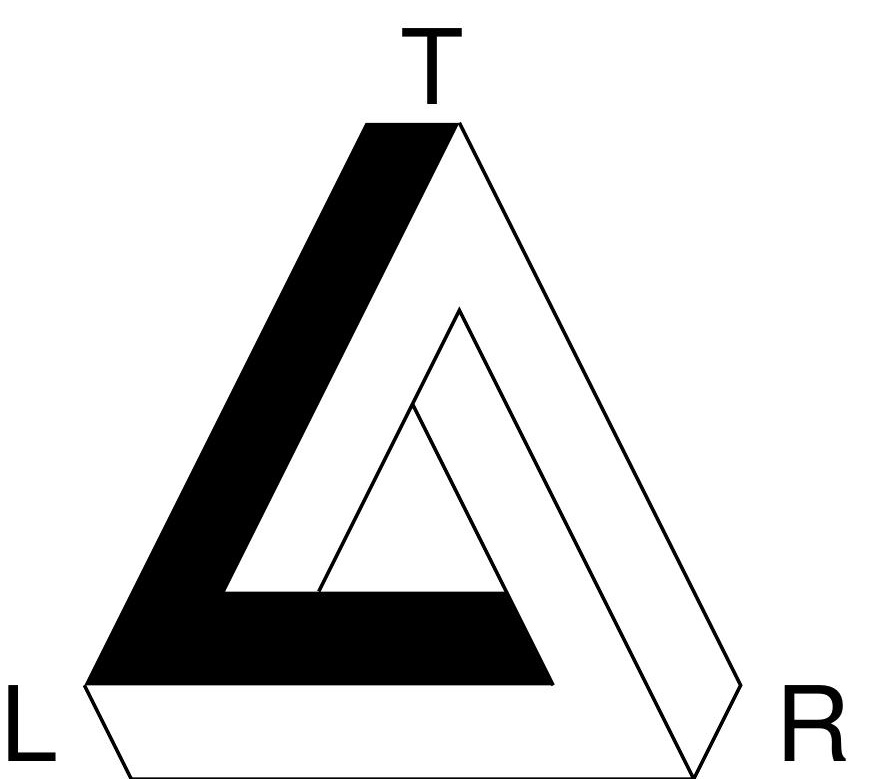

